\title{
Development of Novel Protective Polyvalent Irradiated Pseudomonas Aeruginosa Vaccine for Immuno-Compromised Patients
}

\author{
Manal M.E. Ahmed ${ }^{1,{ }^{*}}$, Jakeen Eljakee ${ }^{2}$, Tarek Mahran ${ }^{3}$ \\ ${ }^{1}$ Pharmacology Department, Medical Research Division, National Research Centre \\ ${ }^{2}$ Microbiology Department, Faculty of Veterinary Medicine, Cairo University \\ ${ }^{3}$ Microbiology Department, Egyptian Atomic Energy Authority (EAEA) \\ *thinktankteam2014@gmail.com
}

Keywords: P. aeruginosa, vaccine, immuno-compromised, irradiation, protective efficacy, challenge test.

\begin{abstract}
Pseudomonas aeruginosa is an opportunistic pathogen affecting immuno-compromised patients; however, no effective vaccine is currently available in the market. Here, we developed novel polyvalent irradiated $P$. aeruginosa vaccine using cobalt 60 that inhibited pathogen viability but retained antigenic expression functionally. Mice were vaccinated by the developed vaccine by intranasal, intramuscular and subcutaneous route of administration followed by challenge test. The protective efficacy of the novel vaccine reached up to $95 \%$. This significant protection was mainly associated with measurable antiserum opsonic killing activity. In conclusion, the novel vaccine provides a promising strategy of both prophylactic and therapeutic approaches for immunocompromised patients against MDR $P$. aeruginosa.
\end{abstract}

\section{Introduction}

Pseudomonas aeruginosa is a Gram negative, ubiquitous opportunistic pathogen. It is responsible on multiple life threatening infections especially in patients with cystic fibrosis and immuno-compromised patients. It is one of the primary causes of bacteremia and sepsis in hospitalized patients $[1,2]$. The wide use of broad-spectrum antibiotics and disinfectants empirically has led to the continuous emergence of multidrug resistant $P$. aeruginosa strains that present a major challenge to clinical therapy and contribute significantly to increased morbidity and mortality worldwide [3]. Also, biofilm formation can further increase its resistance by 1000 fold [4]. One of the promising therapeutic approaches is immunotherapy, which targets $P$. aeruginosa virulence factors to reduce its pathogenesis without inducing multidrug resistance $[5,6]$. The complexity of the $P$. aeruginosa genome, which encodes many antigens, indicates that immunotherapy using a single antigen will not provide sufficient protection. The previous studies revealed that $P$. aeruginosa vaccines that targeting certain pseudomonal virulence factors, such as LPS, O, flagellar antigen [7], the outer membrane proteins F and I [8], or the type III secretion system component PcrV [9], have conventional protective mechanisms, namely, antibody-mediated opsonophagocytic killing and/ or antibody-mediated toxin inhibition. Whoever LPS $\mathrm{O}$ antigen-based vaccines confer high levels of protection, their clinical usage is quite limited. This is because of $\mathrm{O}$ antigen diversity among $P$. aeruginosa isolates [10]. Recently, the studies have been focused mainly on antigens with conserved epitopes. Polyvalent or multivalent vaccines are developed for immunizing against two or more strains of the same microorganism, or against two or more different microorganisms[11]. Polyvalent vaccines demonstrate promising protection. Inactivation using gamma radiation is not a new technique, and has been previously used to sterilize foods and prepare inactivated vaccines. Superiority of gamma radiation over conventional inactivation methods, such as heat and chemical treatments, still a controversial issue. However, because of its ability to penetrate microorganisms effectively and target nucleic acids specifically thus causing less damage to surface antigenic proteins, needs to use of gamma radiation are increasing to develop safe and effective vaccines. In addition to its ability to inactivate large volumes, they can be stored in closed containers, and there is no requirement to remove chemical compounds after inactivation[12]. Here, we prepared polyvalent 
irradiated $P$. aeruginosa vaccine. Our study indicates that the prepared vaccine has a potential protective efficacy.

\section{Materials and Methods}

1. Strains of P. aeruginosa: $P$. aeruginosa standard strain $\mathrm{PAO} 1$ was purchased from ATCC (Manassas, VA, USA) to be used as challenge strain. Clinical strains of six $P$. aeruginosa isolates were collected from Eldemerdash surgery hospital, Ain Shams University, Egypt and five $P$. aeruginosa isolates were from the military hospital, Egypt. All isolates collected were identified by the clinical microbiologists at the hospitals from which the isolates were obtained. We confirmed their identity by previously published criteria[13].

P. aeruginosa strains were cultured in Luria-Bertani (LB) medium (Sigma-Aldrich, Shanghai, Trading Co. Ltd) washed and diluted with sterile phosphate buffer saline (PBS) to an appropriate cell concentration determined spectrophotometrically at $600 \mathrm{~nm}\left(\mathrm{OD}_{600}\right)$.

\section{P. aeruginosa antisera for serotyping:}

Sixteen unabsorbed $P$. aeruginosa antisera (1 to 16) based on the International Antigen Typing Scheme Types[14] were obtained from Institute Pasteur, Paris, France. Serotyping was carried out as described by Legakis et al.[15] (Table 1).

Table 1: Serotyping of the clinical strains of $P$. aeruginosa.

\begin{tabular}{ccc}
\hline $\begin{array}{c}\text { Clinical } \\
\text { Strains } \\
\text { Codes }\end{array}$ & $\begin{array}{c}\text { Lanyi-Bergans } \\
\text { O-serogroups }\end{array}$ & No of isolates \\
\hline $\mathbf{1 7 0 0 0 1}$ & O3 & 3 \\
$\mathbf{1 7 0 0 1 0}$ & O6 & 4 \\
$\mathbf{1 7 0 0 2 2}$ & O15 & 1 \\
$\mathbf{1 7 0 0 2 3}$ & O12 & 2 \\
\hline $\mathbf{1 7 0 0 2 1}$ & O4 & \\
\hline
\end{tabular}

3. Animals: 8 to 12 -week-old female BALB/c mice were purchased from the holding company of vaccines and biological products, VACSERA, Egypt. Mice were matched for age and sex, and kept under specific pathogen - free (SPF) conditions. All animal experiments were complied with institutional animal care committee (IACC) guidelines regarding the use of animals in research.

\section{Preparation of irradiated $P$. aeruginosa vaccine:}

a. Determination of the effective irradiation dose:

Pseudomonas aeruginosa serogroups O3, O4, O6, O12 and $\mathrm{O} 15$ were grown separately in LB agar medium for $24 \mathrm{~h}$ at $37^{\circ} \mathrm{C}$. Lope from each single colony was transferred into LB broth medium for $16-18 \mathrm{~h}$ at $37^{\circ} \mathrm{C}$ to be in logarithmic phase. The culture was centrifuged at $3000 \mathrm{Xg}$ for $15 \mathrm{~min}$. The palettes were re-suspended in PBS and centrifuged again at $3000 \mathrm{Xg}$ for $15 \mathrm{~min}$. Then collected bacteria were adjusted to a concentration of $10^{6} \mathrm{CFU} / \mathrm{ml}$ and lyophilized using LABCONCO Lyophilizer, USA then exposed to various doses of irradiation $(0.5,1.0,1.5,2.0,2.5,3.0,3.5,4.0$, 4.5, 5.0, 5.5, 6.0 Kilo Gray) using Cobalt 60 at escalating intervals at Egyptian Atomic Energy Authority (EAEA) to select the effective dose.

\section{b. Determination of bacterial replicate viability:}

The replication viability of $P$. aeruginosa serogroups were confirmed using plating serial dilutions on LB agar medium. The colony forming units (CFUs) on the bacterial culture plates were manually counted by a technician blinded to the experimental conditions after incubating for 24 hours. 


\section{c. Determination of bacterial replicate metabolic activity:}

The metabolic activity for each serogroup was assayed calorimetrically using Alamar Blue (BioSource International, Camarillo, CA), which incorporated a colorimetric growth indicator based on the detection of metabolic activity. The irradiated serogroups were firstly washed using PBS and then suspended in nutrient agar medium to the original concentration of $10^{6} \mathrm{CFU} / \mathrm{ml} .100 \mu \mathrm{l}$ irradiated suspensions were incubated with $10 \mu \mathrm{l}$ Alamar blue dye for four hours in a 96-well plate. The metabolic activity was determined at $\mathrm{OD}_{600}$ from $\mathrm{OD}_{570}$

\section{d. P. aeruginosa Vaccine preparation:}

Pseudomonas aeruginosa serogroups O3, O4, O6, O12 and $\mathrm{O} 15$ were grown separately in nutrient broth (Sigma) for $16-18 \mathrm{~h}$ at $37^{\circ} \mathrm{C}$. The culture was centrifuged at $3000 \mathrm{Xg}$ for $15 \mathrm{~min}$. The palettes were re-suspended in PBS and centrifuged again at $3000 \mathrm{Xg}$ for $15 \mathrm{~min}$. The collected bacteria were adjusted to a concentration of $10^{6} \mathrm{CFU} / \mathrm{ml}$ and lyophilized using LABCONCO Lyophilizer, USA. The serogroups powder were pooled together with the same quantity then exposed to the dose of irradiation using Cobalt 60 at $3.5 \mathrm{~K}$ Gy.

Quality control tests were applied on the prepared vaccine to confirm its purity, sterility, safety and completion of inactivation. The prepared polyvalent irradiated lyophilized vaccine were then stored at $-20{ }^{\circ} \mathrm{C}$ till vaccination.

\section{Vaccination programs:}

Eighty Balb/c mice were divided into four groups. Group (A) was vaccinated by placing $20 \mu 1$ of the prepared polyvalent irradiated $P$. aeruginosa vaccine inoculums into each nasal $(40 \mu \mathrm{l}$ containing 1 $\times 10^{8}$ CFUs per mouse). Group (B) was vaccinated by $0.5 \mathrm{ml}$ containing $1 \times 10^{8} \mathrm{CFUs}$ of the prepared polyvalent irradiated $P$. aeruginosa vaccine subcutaneously. Group (C) was vaccinated by $0.5 \mathrm{ml}$ containing $1 \times 10^{8}$ CFUs of the prepared polyvalent irradiated $P$. aeruginosa vaccine intramuscularly and Group (D), the control group received equal volume of PBS intramuscularly. All groups were administered four doses at two weeks intervals. For group (B) and group (C), the prepared vaccine was administrated in Complete Freund's Adjuvant (CFA) for the first dose and in Incomplete Freund's Adjuvant (IFA) for the booster doses. Blood samples were collected at day (0) and every two weeks before administration of each dose to assess the humoral responses evoked by the prepared polyvalent irradiated $P$. aeruginosa vaccine.

\section{Evaluation of the humoral immune response post vaccination using Enzyme Linked Immunosorbent Assay (ELISA):}

To determine the levels of specific antibodies in mouse sera, the immunoassay was applied using ELISA plates coated with a whole live cell of $P$. aeruginosa strains PAO1. Briefly, $100 \mu$ of $P$. aeruginosa strain PAO1 was incubated at $37^{\circ} \mathrm{C}$ then overnight at $4{ }^{\circ} \mathrm{C}$, washed with $0.5 \%$ TweenPBS (T-PBS) and blocked using PBS with 3\% bovine serum albumin (Sigma-Aldrich). Serial dilutions of sera from vaccinated and control mice were incubated overnight at $37^{\circ} \mathrm{C}$ for 30 minutes and washed 3times with T-PBS. $100 \mu \mathrm{l}$ of diluted horseradish peroxidase-conjugated goat anti-mouse (HRP; Sigma-Aldrich) was incubated for $1 \mathrm{~h}$. Plates were then washed three times with T-PBS, and $100 \mu \mathrm{l}$ TMB substrate (Sigma-Aldrich) was added per well. After further incubation for 15 minutes titer.

\section{Challenge experiment:}

a. Preparation of $\boldsymbol{P}$. aeruginosa inocula for In Vivo challenge experiment: $P$. aeruginosa strain PAO1 were grown overnight at $37^{\circ} \mathrm{C}$ in LB. The bacteria were diluted 1:100 in fresh medium and grown to an $\mathrm{OD}_{600}$ of 1.0. For intranasal challenge experiments, bacteria were washed using PBS and diluted to the indicated concentration that were determined by plate counting. 


\section{b. Evaluation of the protective efficacy using challenge test:}

At day 56, mice were challenged intranasally with $1 \times 10^{7}$ CFUs of $P$. aeruginosa strain PAO1 directly into each nostril, as mentioned before. Broncho-alveolar lavage fluid (BALF) was collected 6 and $18 \mathrm{~h}$ after infection for quantitation of CFUs. Clinical signs of $P$. aeruginosa were recorded from day 1 to the day 7 after inoculation. Seven days after inoculation, the presence of the challenge organism was investigated by harvesting the lung, liver, blood, and spleen for bacterial load enumeration. A protected mouse was defined as a mouse that showed no clinical signs, no gross lesions at postmortem and failed to yield any challenge organism on culture.

\section{c. Quantitation of CFUs in Broncho-alveolar lavage fluid (BALF):}

Six and eighteen hours after infection with $1 \times 10^{7}$ CFUs of $P$. aeruginosa strain PAO1 directly into each nostril, Broncho-alveolar lavage fluid (BALF) was subjected for quantitation of CFUs.

\section{Statistical analysis}

All data were analyzed using GRAPHPAD PRISM software (GraphPad, San Diego, CA). Data were analyzed using ANOVA (multiple groups), and multiple comparisons between the groups were performed using Newman-Keuls method after ANOVA. Survival data were plotted using KaplanMeier curves and analyzed by the log-rank test. For measurements of bacterial CFUs, groups were compared using a nonparametric Mann-Whitney U-test. $\mathrm{P}<0.05$ was considered to be statistically significant for all experiments. All values were presented as the mean $\pm \mathrm{SD}$, with the exception of bacterial counts, for which median values were designated.

\section{Results}

Evaluation of the effect of Cobalt 60 irradiation on the viability and metabolic activity of $P$. aeruginosa: As shown in Figure (1a), an expected reduction in the viability of $P$. aeruginosa serogroups was observed with the increase in irradiation doses. A complete loss of $P$. aeruginosa replication viability was detected at a minimum irradiation dose of $3.5 \mathrm{~K} \mathrm{~Gy}$. Also, the metabolic activity of all $P$. aeruginosa serogroups reduced with the increase in irradiation dose. At the irradiation dose of $3.5 \mathrm{~K}$ Gy, the metabolic activity was $59.12 \pm 3.47 \%$ comparing with the active live strain (Figure 1b).

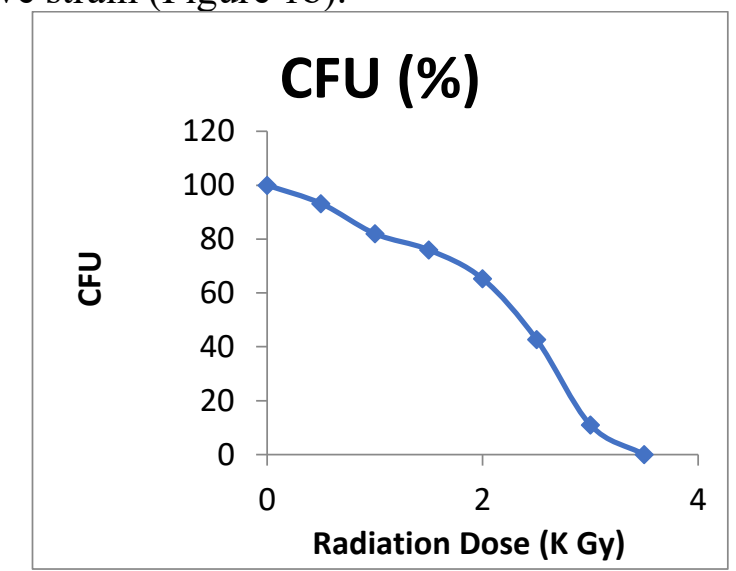

1(a)

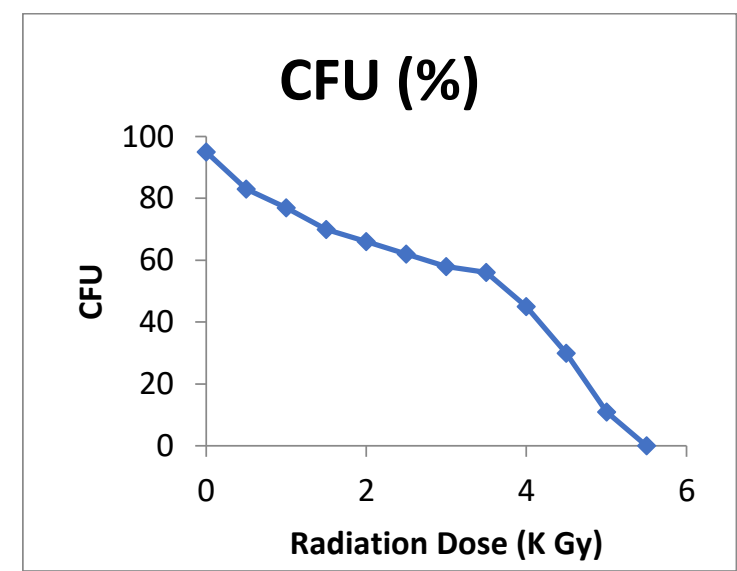

1(b)

Figure 1: The effect of Cobalt 60 radiation dose on the viability and metabolic activity of $\boldsymbol{P}$. aeruginosa: The increase in the radiation dose, the decrease in $P$. aeruginosa reproductive viability that measured by plat counting of CFUs (1a) and metabolic activity that assayed calorimetrically using Alamar Blue (1b). The results are expressed as mean \pm SD. 


\section{Evaluation of the humoral immune response for the prepared polyvalent irradiated $P$. aeruginosa vaccine:}

An increase in serum IgG levels overtime was recorded starting from the second week after the first immunization. The specific antibodies titer was significantly differ according to the route of vaccination. It reached up to $1 / 5120$ among group (A) that vaccinated intranasal and 1/2560 among group (B) and group (C) those vaccinated subcutaneously and intramuscularly, respectively, at two weeks post vaccination program $(\mathrm{P}<005)$ as shown in Figure $(2)$.

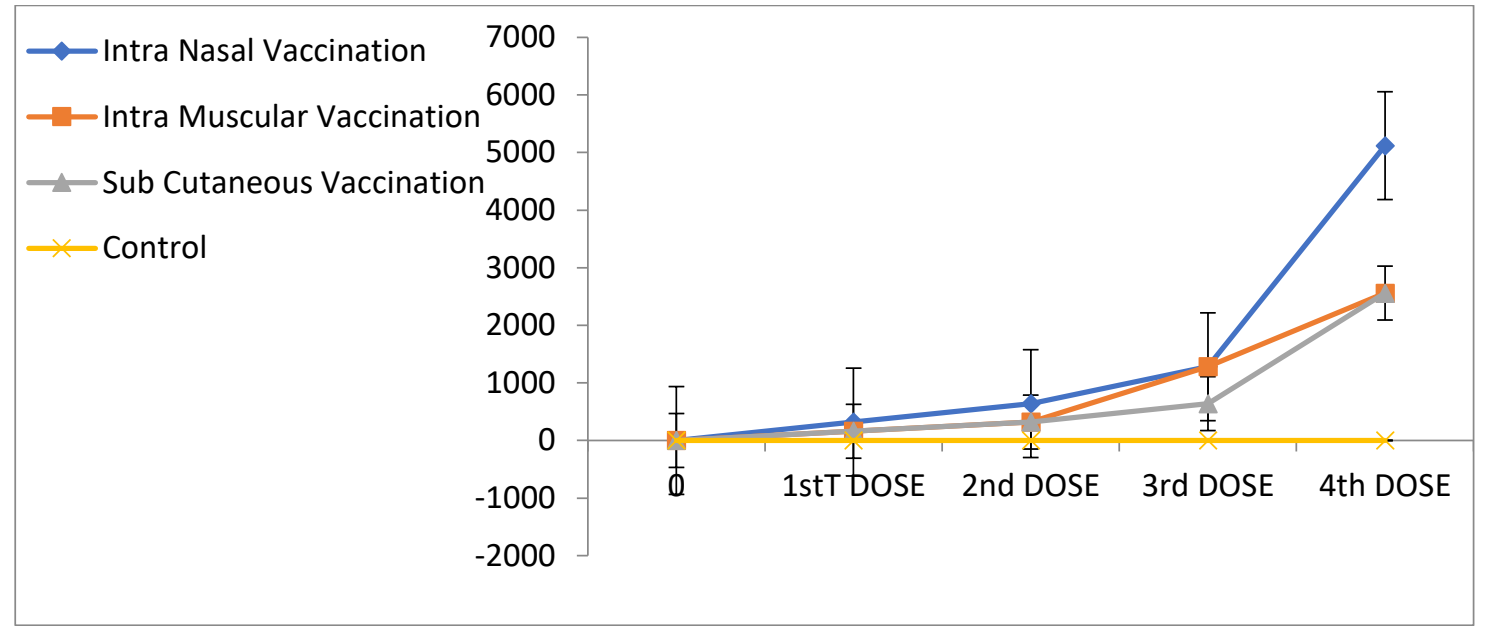

Figure 2: Serum IgG levels among immunized groups using ELISA: Blood samples were collected at day (0) and every two weeks before administration of each dose to assess the humoral responses evoked by the prepared polyvalent irradiated $P$. aeruginosa vaccine. An increase in serum IgG levels overtime was recorded starting from the second week after the first immunization. The specific antibodies titer was significantly differ according to the route of vaccination. Values are represented as mean \pm SD based on five mice in each group $(\mathrm{P}<005)$.

Protective efficacy of the prepared polyvalent irradiated $\boldsymbol{P}$. aeruginosa vaccination in vivo: We found that the prepared vaccine has significant protective effect against lethality of $P$. aeruginosa strain PAO1 $(\mathrm{p}<0.01)$ when compared with the controls after challenged by a relative. Specifically, vaccination led to $95 \%, 85 \%, 90 \%$ protective effect among group (A), group (B) and group (C), respectively (Figure $3 \& 4$ ).

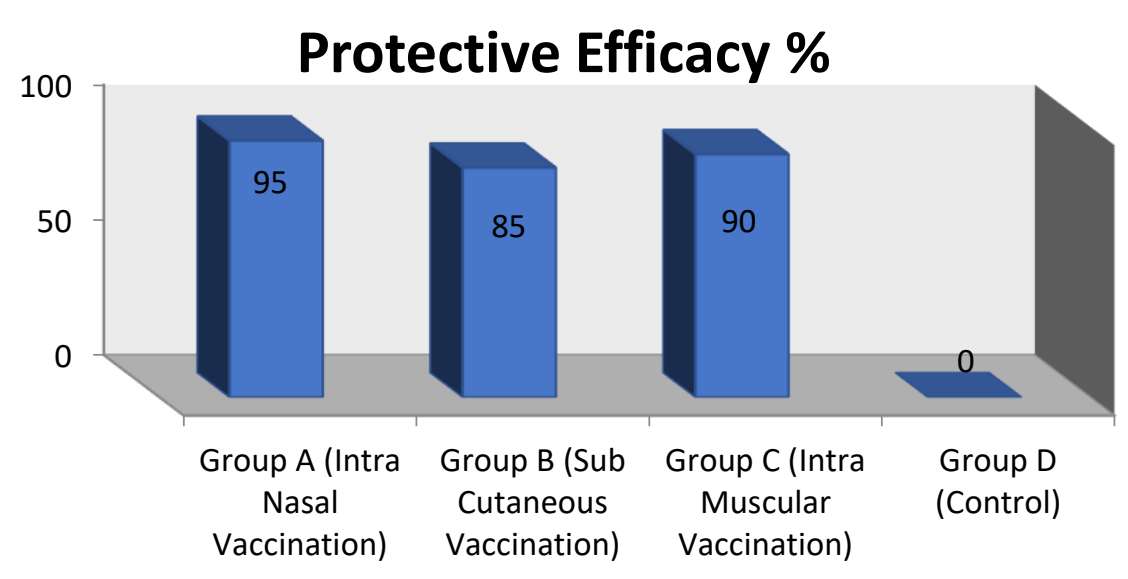

Figure 3: Protective efficacy of the prepared polyvalent irradiated $P$. aeruginosa vaccine in vivo: The vaccinated mice were challenged at day 56 with $1 \times 10^{7}$ CFUs of $P$. aeruginosa strain PAO1 directly into each nostril. Vaccination induced $95 \%, 85 \%, 90 \%$ protective effect among group $(\mathrm{A})$, group $(\mathrm{B})$ and group $(\mathrm{C})$, respectively 


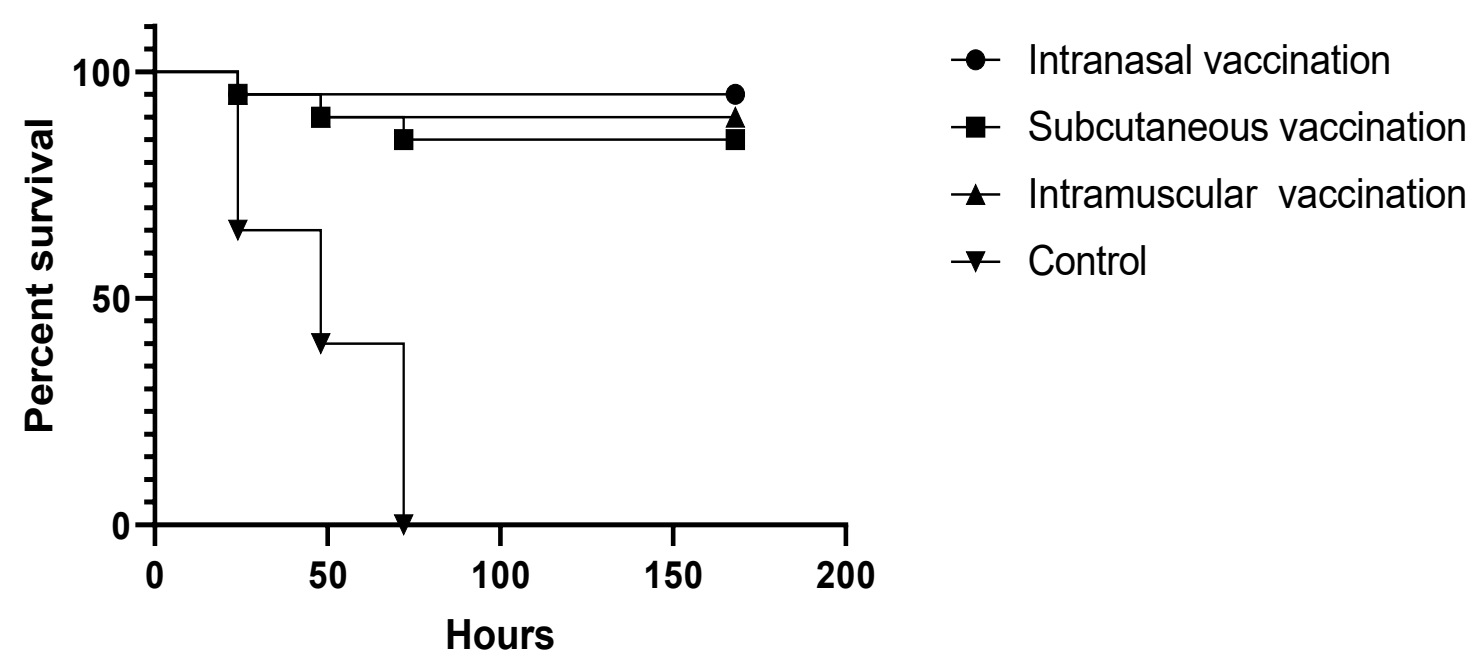

Figure 4: Evaluation of the vaccine induced protection against $P$. aeruginosa in vivo: The vaccinated mice were challenged at day 56 with $1 \times 10^{7}$ CFUs of $P$. aeruginosa strain PAO1 directly into each nostril and monitored the seven days survival rates. Kaplan-Meier curves were plotted for mice of the four groups $(\mathrm{n}=20, \mathrm{P}<0.01)$.

\section{Clinical signs and gross lesions post challenge test:}

The infected cases post challenge were suffering mainly from pneumonia with its characteristic clinical signs including fever and chills, difficulty breathing, cough which sometimes with yellow, green or bloody mucus. Postmortem inspection of died cases was revealed that the presence of severe congestion in the internal organs including lung, liver and spleen. Numbers of cases per group those suffering from $P$. aeruginosa clinical signs and demonstrated colonization post challenge test are listed in Table (2). Titer of $P$. aeruginosa in BALF 6 hours and 18 hours post challenge test was as shown in Table (3).

Table 2: Numbers of cases per group suffering from $P$. aeruginosa clinical signs and demonstrated colonization post challenge test.

\begin{tabular}{lcccc}
\hline \multicolumn{1}{c}{ Groups } & $\begin{array}{c}\text { No. of mice with } \\
\text { clinical signs }\end{array}$ & \multicolumn{2}{c}{ Fate } & \multirow{2}{*}{$\begin{array}{c}\text { No. of mice with } \\
\text { colonization }\end{array}$} \\
\cline { 3 - 4 } & & Died & Recovered & \\
Group A & 2 & 1 & 1 & $\mathbf{2}$ \\
Group B & 5 & 3 & 2 & $\mathbf{7}$ \\
Group C & 5 & 2 & 3 & $\mathbf{6}$ \\
Group D & 20 & 20 & 0 & $\mathbf{2 0}$ \\
\hline
\end{tabular}

Table 3: Titer of $P$. aeruginosa in BALF 6 hours and 18 hours post challenge test.

\begin{tabular}{ccccc}
\hline Vaccinated Groups & \multicolumn{2}{c}{ After 6 hours } & \multicolumn{2}{c}{ After 18 hours } \\
\cline { 2 - 5 } & $\begin{array}{c}\text { Number of } \\
\text { cases }\end{array}$ & CFUs & $\begin{array}{c}\text { Number of } \\
\text { cases }\end{array}$ & CFUs \\
Group A & 2 & $10^{8}$ & 1 & $\mathbf{1 0}^{\mathbf{9}}$ \\
Group B & 5 & $10^{8}$ & 3 & $\mathbf{1 0}^{\mathbf{9}}$ \\
Group C & 5 & $10^{8}$ & 2 & $\mathbf{1 0}^{\mathbf{9}}$ \\
Group D & 20 & $10^{8}$ & 20 & $\mathbf{1 0}^{\mathbf{9}}$ \\
\hline
\end{tabular}




\section{Discussion}

Pseudomonas aeruginosa is a highly complex gram-negative facultative anaerobic pathogen with a wide variety of arsenals to destroy host defense mechanisms. It is one of common causes of nosocomial infections. $P$. aeruginosa infections are mainly associated with immuno-compromised host defense which may include patients with severe burns, diabetes, cancer, organ transplants or immunodeficiency. In lung infection, $P$. aeruginosa opportunistically colonizes patients with cystic fibrosis [16] or chronic obstructive pulmonary disease through disrupting upper and lower airway homeostasis, damaging the epithelium and evading innate and adaptive immune responses [17]. Understanding $P$. aeruginosa pathogensis is essential to know $P$. aeruginosa interactions with the host via flagella, pili lipoproteins, lipopolysaccharides, and the type III secretion system localized in the outer membrane. In addition to, $P$. aeruginosa quorum-sensing molecules regulate the release of soluble factors that enhance the spread of infection. The characteristics of $P$. aeruginosa differentially affect lung epithelial, innate and adaptive immune cells involved in the production of mediators and the recruitment of additional immune cell subsets. The wide range of virulence factors and the potential of $P$. aeruginosa to infect different tissues have made it difficult to determine the main and most important microbial antigen target, thus the development of vaccines for $P$. aeruginosa has been hindered by the complexity of its pathogenesis. Therefore, many $P$. aeruginosa antigens have been tested as vaccine candidates but on the best of my knowledge none of these has demonstrated effective broad protection against multiple strains [18]. Polyvalency has been arguably more useful than using individual immunogen to target multiple Serotypes. Polyvalent vaccines or multivalent vaccines have steadily advanced despite complexity and barriers to manufacturing compared to monovalent vaccines as the manufacture of polyvalent vaccines is associated with more exhaustive quality control measures[19]. Using ionizing radiation such as X-rays and gamma rays, has increased massively over the last 3 decades in the medical and pharmaceutical industry including vaccine production. Radiation technology has many advantages such as removal of the chemical contaminants and penetration of pathogens to damage the DNA specifically thus causing less damage to surface antigenic proteins. However, the production of irradiated vaccines has not been applied widely over the last decades for two main reasons. First, the development of new radiation techniques has been considered impractical or difficult due to issues accessing the radiation equipment. Second, it has been thought that modern subunit vaccines would provide a solution, as they can be developed more easily [20]. The recent successful development of irradiated vaccines for human malaria and influenza have demonstrated the feasibility and practicality of this technique, and have shown that technical problems can be overcome using existing expertise, without needing to resort to sophisticated technology[21, 22]. Furthermore, this technology has been used to produce an anti-cancer vaccine by inactivating cancer cells[23]. Our data suggested that inactivation of $P$. aeruginosa with irradiation could provide cross protective immunologic response for wide spectrum of $P$. aeruginos $a$ serogroups which is not limited to LPS $\mathrm{O}$ antigen.

Here, we prepared polyvalent irradiated Pseudomonas aeruginosa vaccine that containing serogroups O3, O4, O6, O12 and O15 using Cobalt 60 at 3.5 K Gy. Our trails revealed that 3.5 K GY was the minimum irradiation dose that induced a complete loss of $P$. aeruginosa replication viability while the metabolic activity was $59.12 \pm 3.47 \%$ comparing with the active live strain. Quality control tests including purity, sterility, safety and completion of inactivation were applied on the prepare vaccine before using in vaccination program. We performed our experiment where the prepared vaccine was given in four doses at two weeks intervals intranasally, subcutaneously and intramuscularly.

Most bacterial infections can stimulate both innate and adaptive immune responses [24]. $P$. aeruginosa is one of extracellular pathogens, therefore humoral, mucosal or systemic opsonizing immunity is most effective preventing bacterial colonization and subsequently infection [25, 26]. Many studies have been reported that the most important immune effector for most $P$. aeruginosa vaccines was serum opsonic antibody directed against the LPS O antigen. Also, T lymphocyte responses have been reported to mediate important protective immunity among infected persons with $P$. aeruginosa [27 - 29]. From all of the above effective resolution of $P$. aeruginosa infection or its 
clearance is mediated by a combination of neutralizing specific antibodies and inflammatory responses of activated macrophages, neutrophils and other immune cells.

So, an ideal vaccine should comprise a broad range of antigens to elicit both humoral and cellular immunogenic responses. In our study, significant protection was achieved with the prepared polyvalent irradiated $P$. aeruginosa vaccine that was mainly associated with measurable antiserum opsonic killing activity. Blood samples were collected at day (0) and every two weeks before administration of each dose for evaluation of the humoral immune response post vaccination using ELISA. The specific antibodies titer was significantly differ according to the route of administration. It reached up to $1 / 5120$ among group (A) that vaccinated intranasally and 1/2560 among group (B) and group $(\mathrm{C})$ those vaccinated subcutaneously and intramuscularly at two weeks post vaccination program $(\mathrm{P}<005)$.

The vaccination/challenge trail was the golden standard test to evaluate the protective efficacy of the prepared polyvalent irradiated $P$. aeruginosa vaccine. Therefore, vaccinated mice were challenged at day 56 with $1 \times 10^{7}$ CFUs of $P$. aeruginosa strain PAO1 directly into each nostril. We found that the prepared vaccine has significant protective effect against lethality of $P$. aeruginosa strain PAO1 $(\mathrm{P}<$ $0.05)$ when compared with the controls after challenged by a relative as vaccination induced $95 \%$, $85 \%, 90 \%$ protective effect among group (A), group (B) and group (C), respectively. We maintain optimism that an effective $P$. aeruginosa polyvalent irradiated vaccine may eventually be developed and tested in humans toward reducing the morbidity and mortality associated with lung infections caused by $\mathrm{P}$. aeruginosa.

\section{Conclusion}

We can concluded that irradiation of whole $P$. aeruginosa proves to be a safe and effective vaccine strategy in our preclinical study. Our findings might provide a novel strategy of prophylactic and therapeutic vaccine preparation against $P$. aeruginosa. This novel vaccine indeed obtained a better pathogens clearance effect. Moreover, this strategy also saves the time and resources of identifying potential antigenic components for preparation of subunit vaccines. The availability and rapidity of irradiation would expedite vaccine deployment for mass vaccination. Further studies are still required to determine its cellular immunogenic responses and also to establish this strategy in the physician tool box for human infections.

\section{Acknowledgements}

We thank the department of microbiology, Eldemerdash surgery hospital, Ain Shams University, Egypt and the department of Microbiology, the military hospital, Egypt, for providing us with $P$. aeruginosa strains.

\section{References}

[1] P. H. Gilligan, Infections in patients with cystic fibrosis: diagnostic microbiology update, Clinics in Laboratory Medicine. 34(2) (2014) 197-217.

[2] N. Safdar, C. Dezfulian, H. R. Collard, S. Saint, Clinical and economic consequences of ventilator-associated pneumonia: a systematic review, Critical Care Medicine. 33(10) (2005) 2184-2193.

[3] B. K. Chan, M. Sistrom, J. E. Wertz, K. E. Kortright, D. Narayan, P. E. Turner, Phage selection restores antibiotic sensitivity in MDR Pseudomonas aeruginosa, Scientific Reports. 6 (2016) 26717.

[4] A. Brooun, S. Liu, K. Lewis, A dose-response study of antibiotic resistance in Pseudomonas aeruginosa biofilms. Antimicrob Agents Chemother. 44 (3) (2000) 640-646. 
[5] M.W. Douglas, K. Mulholland, V. Denyer, T. Gottlieb, Multi-drug resistant Pseudomonas aeruginosa outbreak in a burns unit - an infection control study, Burns: Journal of the International Society for Burn Injuries. 27( 2) (2001) 131-135.

[6] M. Mudau, R. Jacobson, N. Minenza, L. Kuonza, V. Morris, H. Engelbrecht, M. P. Nicol, C. Bamford, Outbreak of multidrug resistant Pseudomonas aeruginosa bloodstream infection in the haematology unit of a South African academic hospital, PLoS One. 8(3) (2013) e55985.

[7] G. B. Pier, Promises and pitfalls of Pseudomonas aeruginosa lipopolysaccharide as a vaccine antigen, Carbohydrate Research. 338(23) (2003) 2549-2556.

[8] E.T. Weimer, H. Lu, N.D. Kock, D.J. Wozniak, S. B. Mizel, A fusion protein vaccine containing Opr F epitope 8, OprI, and type A and B flagellins promotes enhanced clearance of nonmucoid Pseudomonas aeruginosa, Infection and Immunity. 77 (6) (2009) 2356-2366.

[9] T. Sawa, T. L. Yahr, M. Ohara, K. Kurahashi, M. A. Gropper, J. P. Wiener-Kronish , D. W. Frank, Active and passive immunization with the Pseudomonas V antigen protects against type III intoxication and lung injury, Nature Medicine. 5 (4) (1999) 392-398.

[10] S. L. Gellatly, R. E. Hancock, Pseudomonas aeruginosa: new insights into pathogenesis and host defenses, Pathog Dis. 67 (2013) 159 -173.

[11] Polyvalent vaccine at Dorlands Medical Dictionary Archived March 7, 2012, at the Wayback Machine

[12] H. S. Seo, Application of radiation technology in vaccines development, Clin. Exp. Vaccine Res. (2015).

[13] R. Hugh and G. L. Gilardi, 1980. Pseudomonas, P. 288 - 317. In E. H. Lennette, A. Balows, W. J. Hauler, Jr., and J. P. Truant (Ed.), Manual of clinical Microbiology, 3rd ed. American Society for Microbiology, Washington, D. C.

[14] T. Bergan, 1975, Epidemiological typing of Pseudomonas aeruginosa, P. 189-235. In M. R. BBrown (ed.) Resistance of Pseudomonas aeruginosa. John Wiley \& Sons, Inc., New York.

[15] N.J. Legakis, M. Aliferopoulou, J. Papavassiliou, and M. Papapetropoulou, Serotypes of Pseudomonas aeruginosa in clinical specimens in relation to antibiotic susceptibility. J. Clin. Microbiol. (1982),16(3): 458-463.

[16] N. Mayer-Hamblett, M. Rosenfeld, R. L. Gibson, B. W. Ramsey, H. D. Kulasekara, G. Z. Retsch-Bogart, W. Morgan, D. J. Wolter, C. E. Pope, L. S. Houston, B. R. Kulasekara, U. Khan, J. L. Burns, S. I. Miller, L. R. Hoffman, Pseudomonas aeruginosa in vitro phenotypes distinguish cysticfibrosis infection stages and outcomes, Am J Respir Crit Care Med. 190(2014) 289-297.

[17] T.F. Murphy, A.L. Brauer, K. Eschberger, P. Lobbins, L. Grove, X. Cai, S. Sethi, Pseudomonas aeruginosa in chronic obstructive pulmonary disease, Am J Respir Crit Care Med. 177 (2008) 853-860.

[18] G. P. Priebe, J. B. Goldberg, Vaccines for Pseudomonas aeruginosa: a long and winding road, Expert Rev Vaccines. 13 (2014) 507-519.

[19] J. Smith J, M. Lipsitch, J. W. Almond, Vaccine production, distribution, access and uptake. Lancet. 378(2011) 428-38.

[20] Plotkin SA. History of vaccine development. New York: Springer; 2011.

[21] R. Gasparini, D. Amicizia, P. L. Lai, D. Panatto, Live attenuated influenza vaccine: a review. J Prev Med Hyg. 52 (2011)95-101.

[22] C. Arama, M. Troye-Blomberg, The path of malaria vaccine development: challenges and perspectives. J Intern Med. 227 (2014) 456-466. 
[23] D. T. Le, A. Wang-Gillam, V. Picozzi, et al., Safety and survival with GVAX pancreas prime and Listeria Monocytogenes-expressing mesothelin (CRS-207) boost vaccines for metastatic pancreatic cancer. J Clin Oncol. 33 (2015) 1325-1333.

[24] R. J. Napier, E. J. Adams, M. C. Gold, D. M. Lewinsohn, The Role of Mucosal Associated Invariant T Cells in Antimicrobial Immunity, Front Immunol. 6 (2015) 344.

[25] B. Tummler, L. Wiehlmann, J. Klockgether, N. Cramer, Advances in understanding Pseudomonas, F1000Prime Rep. 6 (2014) 9.

[26] N. Mesaros, P. Nordmann, P. Plésiat, M. Roussel-Delvallez, J. Van Eldere , Y. Glupczynski, Y. Van Laethem, F. Jacobs, P. Lebecque, A. Malfroot, P. M. Tulkens, F. Van Bambeke, Pseudomonas aeruginosa: resistance and therapeutic options at the turn of the new millennium, Clin Microbiol Infect. 13 (2007) 560 -578.

[27] M.L. Dunkley, R.L. Clancy, A.W. Cripps, A role for CD4 + T cells from orally immunized rats in enhanced clearance of Pseudomonas aeruginosa from the lung, Immunology. 83 (1994) 362 -369 .

[28] M.L. Dunkley, A.W. Cripps, P.W. Reinbott, R.L. Clancy, Immunity to respiratory Pseudomonas aeruginosa infection: the role of gut-derived $\mathrm{T}$ helper cells and immune serum, Adv Exp Med Biol. 371B (1995) 771- 775.

[29] M. M. Stevenson, T. K. Kondratieva, A. S. Apt, M. F. Tam, E. Skamene, In vitro and in vivo T cell responses in mice during bronchopulmonary infection with mucoid Pseudomonas aeruginosa, Clin Exp Immunol. 99 (1995) 98-105. 